\title{
Autism, Adverse Events, and Trauma
}

\author{
Ella Lobregt-van Buuren ${ }^{1}$ - Marjolijn Hoekert ${ }^{2}$ - Bram Sizoo ${ }^{1}$ \\ ${ }^{1}$ Dimence Institute of Mental Health, Deventer, The Netherlands; ${ }^{2}$ Mental Health Service \\ Organization GGZ Noord-Holland-Noord, Heiloo, The Netherlands
}

Author for correspondence: Ella Lobregt- van Buuren, Dimence, Deventer,

The Netherlands. E-mail: e.lobregt@dimence.nl

Doi: https://doi.org/10.36255/exonpublications.autismspectrumdisorders.2021.trauma

\begin{abstract}
Clinical and scientific data show a higher risk of adverse events and trauma in people with an autism spectrum disorder (ASD). These experiences are, however, often undiagnosed and untreated in autism. Diagnosing trauma- and autism-related symptoms is complicated due to the overlap between these two. Several pitfalls and points of attention can be mentioned. This chapter highlights the importance of recognizing trauma-related symptoms at an early phase and start of trauma treatment. It also provides an overview of the current knowledge about the feasibility and effectiveness of treatment of trauma-related symptoms in people with ASD. Simultaneous training of self- and emotion regulation skills, taking into account autistic features, is recommended.
\end{abstract}

Keywords: early recognition; emotion regulation; masking; post-traumatic stress disorder; trauma; underdiagnosis

In: Autism Spectrum Disorders. Grabrucker AM (Editor). Exon Publications, Brisbane, Australia. ISBN: 978-0-6450017-8-5; Doi: https://doi.org/10.36255/exonpublications. autismspectrumdisorders. 2021

Copyright: The Authors.

License: This open access article is licenced under Creative Commons Attribution-NonCommercial 4.0 International (CC BY-NC 4.0) https://creativecommons.org/licenses/by-nc/4.0/ 


\section{INTRODUCTION}

Adverse events, trauma and their consequences are frequently overlooked in people with autism spectrum disorders (ASD). At the same time, people with ASD are at increased risk of repeatedly experiencing such events (1), leading to underdiagnosis and undertreatment of trauma-related symptoms. Conversely, emergent co-morbid problems may mask autism-related symptoms. This chapter provides information about prevalence of exposure to adverse events, trauma, and posttraumatic stress disorder (PTSD), and discusses possible explanations for overlooking of adverse events and trauma in people with ASD. Throughout this chapter, the term 'trauma' will refer to the DSM-5 definition of trauma and requires "actual or threatened death, serious injury, or sexual violence" (2). The description of adverse events refers to experiences that can result in PTSD symptoms but does not meet the criteria for PTSD.

There is a growing body of research that pertains to the prevalence of exposure to adverse events and trauma in children with ASD, and the consequences in terms of physical and mental health (3, 4). For example, in a population-based study, it was found that children with ASD reported a significantly higher level of exposure to neighborhood violence, parental divorce, traumatic loss, poverty, mental illness and substance abuse in the family. These situational indicators of stress and trauma experienced by the family are called adverse childhood experiences (ACE) and the probability of reporting one or more of them was higher in children with ASD compared to children without ASD (3).

This difference is especially pronounced in lower income families (5). Also, adults with ASD are exposed to a significantly greater number of adverse events in comparison with individuals without ASD. This has been described as a vulnerability to negative life experiences, which is suggested to be an important factor in the development of co-morbid mental health conditions such as anxiety and depression symptoms (6). Common adverse events with a major impact are loss of study and work, and bullying $(6,7)$. In clinical practice, we see a chronic mismatch between the needs and possibilities of the person with autism, as well as difficulties facing up to the expectations of family and treatment providers. Individuals in whom autism is diagnosed at a later age are most at risk of these chronic mismatch (8).

Exposure to adverse events and trauma does not automatically result in PTSD and has been found to be associated with many other forms of psychopathology, with the strongest link to anxiety and depression $(6,9)$. Anxiety and mood symptoms were found in 50-70\% of children and adults with ASD $(10,11,12)$. One study showed that nearly $90 \%$ of youth with ASD and clinical-level mood symptoms reported at least one trauma, compared to $40 \%$ of those without mood symptoms (13). Adverse events partially explain the higher rates of anxiety and depression symptoms and lower life satisfaction in adults with ASD compared to non-autistic adults (6). Thus, in individuals with ASD there seems to be a strong association between exposure to adverse events and trauma and the presence of anxiety and depressive symptoms.

A proportion of people who experience trauma develops symptoms appropriate to post-traumatic stress disorder (PTSD). In children and adolescents with autism, PTSD appears to occur at a similar or higher rate compared to the general 
population (7). Adults with ASD are more than four times likely to be diagnosed with PTSD than adults without ASD (6). Thus, there are indications of a higher prevalence of exposure to traumatic events and post-traumatic stress disorder (PTSD) in children and adults with ASD. Children and adults with ASD and intellectual disabilities are especially at risk for exposure to adverse events and trauma (14).

Above-mentioned issues regarding trauma and ASD are illustrated using a case study that follows below.

\section{CASE STUDY}

Beline is a 25-year-old woman with autism. She has been seeing social workers since the age of 13 and has followed various (clinical) treatments aimed at mood and anxiety symptoms and eating problems. She was diagnosed with ASD only recently. She lives with her parents and brother and has had no further education or work after graduating from high school. She always felt excluded by other pupils at school. At the time of her admission to a specialized outpatient autism clinic when she was 25 years old, mood and anxiety symptoms were prominent. She also had a negative and empty self-image. In daily life, she experiences problems around daily activities, social contacts, and home situations.

\section{AUTISM SPECTRUM DISORDER AND TRAUMA}

Traditionally, autism has been associated with three cognitive styles: a limited theory of mind, weak central coherence, and executive functioning disorders. Recently, however, the enactive approach for autism, coined by Klin in 2003 (15), has shown to be helpful in understanding where difficulties originate from. The enactive approach has its origins in phenomenology and encourages one to view the world as the individual with autism experiences it (16). This requires exploring how someone makes sense of what goes on around him and how that subsequently directs his activities. Sense-making depends to a certain extent on innate qualities like sensory processing (17). For example, when the neurological threshold is low, sounds may be experienced as painful by individuals with autism, which influences the sense-making process. The way people with autism understand the world can therefore differ from the shared opinion, which may provoke negative feedback, conflict, or ridicule. This, in turn, will influence the way the person with autism regulates his activities in the environment, which will manifest in behavior. In other words, behavior as we see it originates from the phenomenological experience of the world, which may differ from that of persons without autism. This explains why events that would generally not be considered as traumatic may impact as such in people with autism.

Experiencing adverse events and trauma more frequently, combined with a genetic vulnerability when it comes to self-regulation and sense-making, make people with autism more susceptible to the psychosocial consequences of experiencing adverse events (18). Wood and Gadow (19) hypothesized that 
autism-related sensory hyperreactivity to everyday stimuli, social confusion, misunderstanding, and rejection by others might lead to baseline elevated anxiety levels, affecting resilience to cope with stressors. A possible consequence is a severe overload in (young) adults with autism and their family and friends. This can lead to a range of additional problems in the areas of attachment, identity, anxiety, and mood symptoms and subsequently a maladaptive way of regulating themselves through, for example, compulsive behavior, restrictive eating, socially isolating themselves and/or substance use.

\section{UNDERDIAGNOSIS AND UNDERTREATMENT}

As mentioned before, certain stressful events, such as bullying, do not meet the definition of trauma as described in the DSM-5. Patients reporting these experiences are not formally diagnosed with PTSD, and mostly trauma treatment is not provided. In addition, because of a different sense-making, negative events, which people without autism merely see as unpleasant, can be experienced as upsetting or even traumatic by people with autism. Conversely, disturbing or traumatic events experienced by people without autism may not be experienced as traumatic in people with autism (13). Also, people with autism do not always recognize when situations are unsafe, and they are less inclined to share their experiences spontaneously with others (20). These factors increase the risk of underdiagnosis and under-treatment of traumatic symptoms in people with ASD. This is compounded by a phenomenon called diagnostic overshadowing: symptoms attributed to autism may be stress reactions to traumatic or other adverse events. For example, hyperarousal (a symptom of PTSD) overlaps with autism-related sensory hyperreactivity. Feelings of detachment from others (a symptom of PTSD) partly overlap with deficits in social-emotional reciprocity associated with autism. An impaired ability to mentalize and recognize emotions is observed in both people with PTSD and in people with autism. In conclusion, trauma, adverse events, stressful events and trauma-related symptoms may be overlooked or overshadowed by autistic features or other psychopathology and therefore remain untreated. Conversely, autistic features may also long be overlooked or overshadowed by co-morbid problems such as trauma-related symptoms.

If the impact of traumatic events is recognized, however, there is still a risk of undertreatment due to insufficient availability of therapists who understand trauma and autism. If the impact of traumatic events is only recognized at an adult age, there are often serious additional problems in (young) adults. Because of this, trauma treatment becomes more difficult or is not seen as feasible, which leads to a vicious circle.

\section{TREATMENT METHODS}

Little is known about the feasibility and potential effectiveness of treatment of trauma in individuals with ASD and trauma-related symptoms. This may partly be caused by the problem of overlooking and overshadowing as mentioned above. 
Another impeding factor may be that people with ASD are often excluded from participating in research (21). This section gives an overview of what is known about treatment of trauma in autistic children and adults with and without intellectual disabilities.

Eye Movement Desensitization and Reprocessing (EMDR) therapy, Cognitive Behavioral Therapy (CBT) with a trauma focus, and Prolonged Exposure are strongly recommended for the treatment of adults with PTSD. Secondly, standard recommendations for the treatment of adults with PTSD are CBT without a trauma focus, guided internet-based CBT with a trauma focus, Narrative Exposure Therapy and Present-Centred Therapy. Emerging evidence comes from newer interventions such as Virtual Reality Therapy (22).

EMDR therapy is an individual therapy originally designed to alleviate the negative feelings associated with the traumatic memories (23). During EMDR sessions the patient attends to emotionally disturbing material in brief sequential doses while at the same time focusing on external stimuli. Distracting stimuli can vary from the most common used lateral eye movements to a variety of other stimuli including audio stimulation, hand tapping, and making calculations. Shapiro suggested that EMDR treatment facilitates the access to the traumatic memory network so that information processing is enhanced, while new associations are forged between the traumatic memory and more adaptive memories or information. The internal and external triggers are desensitized and thereby trauma related symptoms are alleviated (23).

Data on the application and effectiveness of trauma treatment in children and adults with autism are scarce. Preliminary findings from a few case reports suggest EMDR therapy for PTSD to be feasible and potentially effective for children and adults with ASD also in case of comorbid intellectual disabilities $(24,25)$. Controlled studies are lacking. Only one controlled study has been published that describes the feasibility and efficacy of EMDR for trauma-related symptoms in adults with autism (8). Results showed a significant reduction of symptoms of post-traumatic stress (measured with the Impact of Event Scale-Revised, IES: $\mathrm{d}=1.16$, Figure 1), and a variety of other mental health problems among which severity of autistic features (measured with the Social Responsiveness Scale-Adult version, SRS-A: $\mathrm{d}=0.39$, Figure 2 ). The positive results were maintained at follow-up. The results suggest EMDR therapy added to TAU (treatment as usual) to be a feasible and potentially effective treatment for individuals with ASD who suffer from the consequences of exposure to distressing events. It should be noted that the study was based on a small sample of participants $(n=21)$.

The significant reduction of autistic features concerning social motivation and communication following EMDR therapy, and at follow-up, albeit with a small effect size, is remarkable. A possible explanation for this finding might be that the clinical manifestation of autistic symptoms decreases when individuals with ASD experience less trauma-related stress and psychological distress. Hence, it is conceivable that trauma-related symptoms are a moderator for the severity of ASD symptoms, such that exposure to trauma and other adverse events exacerbate autistic core features. Another possible explanation for the observed reduction of autistic features in the study is diagnostic overshadowing: symptoms of PTSD can be masked by symptoms of autism, therefore symptoms of PTSD, previously seen as features of ASD, might have declined as a result of EMDR therapy (8). 


\section{IES-R}

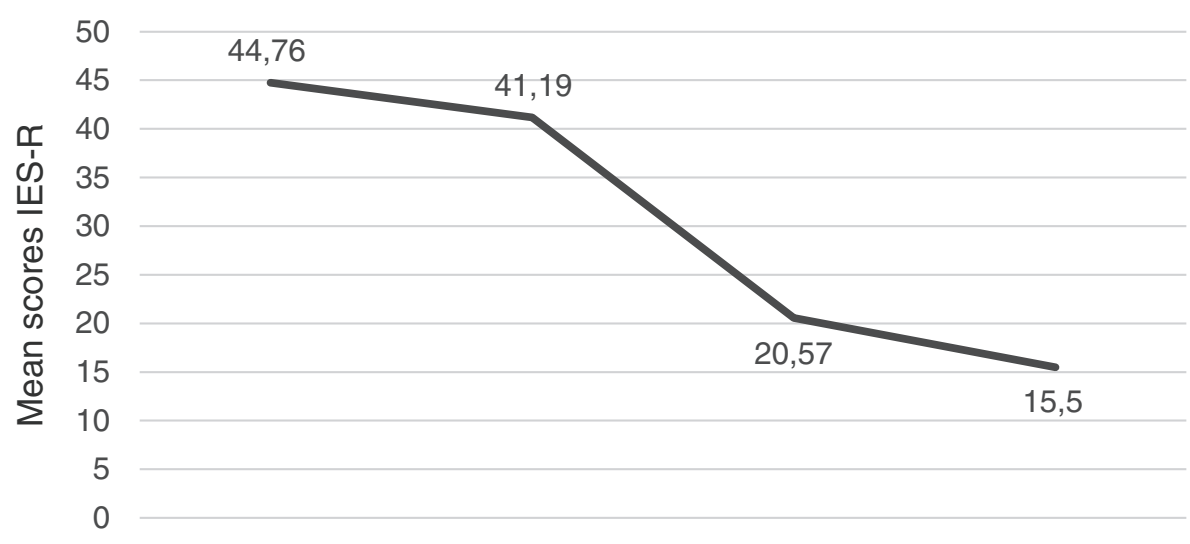

T1: baseline T2: start EMDR T3: end EMDR T4: follow-up

Figure 1. Mean scores IES-R at four moments in time. Post-traumatic stress burden measured with the Impact of Event Scale-Revised (IES-R). T1, 6-8 weeks before, and T2, at the start of EMDR treatment. T3, End of EMDR treatment (weekly for eight weeks), and T4, after 6-8 weeks follow up period.

\section{SRS-A}

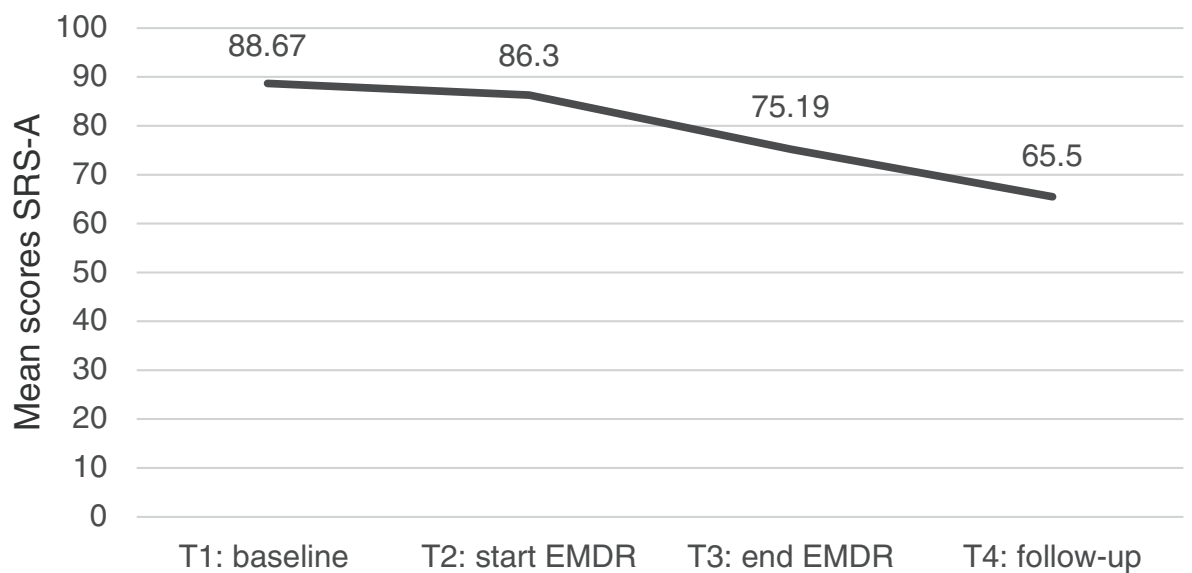

Figure 2. Mean scores SRS-A at four moments in time. The level of self-reported problems in social communication and interaction, measured with the Social Responsiveness Scale-Adult version (SRS-A). T1, 6-8 weeks before, and T2, at the start of EMDR treatment. T3, End of EMDR treatment (weekly for eight weeks), and T4, and after 6-8 weeks follow up period. 
CBT with a Trauma Focus (CBT-T) includes all treatments aiming to alleviate trauma related symptoms by addressing and changing the thoughts, beliefs, and behavior. CBT-T includes doing homework, psycho education, and doing behavioral experiments such as exposure and working on stress management (22). For the treatment of children with PTSD it has shown to be important to involve the caregivers to strengthen caregiver-child relationships (22). Modified versions of CBT interventions may result in reduced trauma symptoms in people with ASD (26). Preliminary recommendations of adapting current trauma focused cognitive behavioral therapy for patients with ASD are provided (26). The most important adaptions are to limit assessment and interventions relying on verbal language and abstract reasoning. Reliance on the verbal report of a patient with ASD during exposure to a traumatic event may be challenging because of a different sense making. It is important to use multiple reporters (e.g., caregivers, parents). Providing concrete descriptions can be helpful and combining oral speech with visual representations. Furthermore, attention should be paid to generalizing new skills in different settings (26).

Prolonged Exposure is a type of CBT during which the patient with PTSD is helped to confront his traumatic memory by using a verbal narrative technique that involves detailed retelling of the traumatic experience that is then recorded and listened to on a repeated basis with the goal of habituation (22). This is combined with real life (in vivo) exposure to fear-evoking situations, also with the aim of habituation. It is recommended to make a step-by-step plan in a structural way. External rewards are essential when conducting in vivo exposure with people with ASD (26). It may be necessary to modify the rating scale to measure distress during exposure, e.g., by providing clear examples of different ratings along with personalized cues. The therapist may also rate the severity of the symptoms during the exposure (26).

Narrative Exposure Therapy (NET) is based on CBT. The therapist guides the patient in the development of a chronological, autobiographical narrative of his life including the most significant positive and negative events. Emotional processing is facilitated by using CBT techniques. Especially the difficult moments, such as traumatic events will be discussed in detail. The aim is to process overwhelming experiences and alleviate trauma related symptoms (22). There are, however, no research data available about NET in adults with autism.

Present-Centred Therapy, recommended as the second best in the treatment of PTSD symptoms, targets daily challenges related to trauma symptoms. It consists of psycho-education about PTSD symptoms and its effect on daily life, training effective strategies to deal with daily challenges and homework to practice new skills (22). No research data are available about Present-Centred Therapy in adults with autism. From clinical practice it is however known that identifying daily challenges and developing self- management skills are essential elements of the treatment of people with ASD.

\section{CASE STUDY: TREATMENT COURSE}

Beline's outpatient treatment started with psycho-educational therapy. Subsequently, the psychological treatment focused on emotion regulation, 
including affect-education, training in the use of a phased crisis plan, and reducing anxiety. During the treatment, the severity and complexity of her complaints became increasingly clear, and it became more difficult to include her surroundings in this. Never-mentioned negative experiences came up during the treatment sessions, such as bullying, sexual assault, and experiences of confinement during earlier admissions. A start was made with EMDR. Because of Beline's difficulty in putting her experiences into words, the therapist and Beline decided to visualize her experiences by making drawings of them. After the first EMDR sessions, the tension increased enormously. At that time, there were several changes and disappointments. Suicidality, which could not be accurately assessed, played a role in deciding that the EMDR treatment in an outpatient setting could not be continued. Admission to an enclosed ward of a general psychiatric clinic followed. Since then, it has not been possible to find a suitable treatment setting for Beline.

At the general psychiatric clinic, most of the team members did not have any experience with working with people with an ASD. Furthermore, the noisiness and unstructured daily rhythm at the clinic had a negative effect on Beline's status and her suicidal behavior increased. Not only Beline but also the nurses were overloaded which resulted in moving from clinic to clinic.

\section{POINTS OF ATTENTION AND PITFALLS IN THE DIAGNOSIS AND TREATMENT OF TRAUMA IN AUTISM}

Trauma history and associated trauma-related symptoms should be routinely questioned at the diagnostic stage in people with autism, considering their limitations in social communication. That what is not explicitly asked often remains undisclosed. A helpful instrument is the Diagnostic Interview Trauma and Stressors for individuals with mild intellectual impairment, (DITS-LVB [Dutch], (27) because of the concrete, visualized, and structured way in which potentially traumatic events and trauma-related symptoms are questioned.

Once severe impairments in self- and emotion regulation are present, like suicidal and self-harming behavior, it is crucial to train adaptive regulation skills systematically before and during trauma treatment. Dialectical Behavior Therapy offers a treatment philosophy for this purpose that is currently being scientifically investigated in adults with autism. An autonomy-promoting policy that is unconditionally supported by the patient, family, and care system is essential because of a risk of parallel processes, such as the repetition of overly exclusive relationships with the patient or the constant taking over of control. Therefore team-wide consensus about treatment policy is relevant. Without paying special attention to this, treatment lines and teams can fall apart.

\section{CONCLUSION}

Early recognition of traumatic experiences and early treatment of their consequences is very important. Particularly in the case of late recognition and treatment, training of self- and emotion regulation skills is crucial in addition to 
the treatment of trauma in a context that considers several aspects of autism. The different forms of treatment of trauma described in this chapter are needed to be further investigated in people with ASD. This can be helpful to improve our understanding of the factors that play a role in the treatment of choice.

Conflict of interest: The authors declare no potential conflicts of interest with respect to research, authorship, and/or publication of this manuscript.

Copyright and permission statement: The authors confirm that the materials included in this chapter do not violate copyright laws. Where relevant, appropriate permissions have been obtained from the original copyright holder(s), and all original sources have been appropriately acknowledged or referenced.

\section{REFERENCES}

1. Hoover DW. The effects of psychological trauma on children with autism spectrum disorders: A research review. Rev J Autism Dev Disord. 2015(2):287-299. https://doi.org/10.1007/s40489-015-0052-y

2. American Psychiatric Association. Diagnostic and statistical manual of mental disorders, DSM-5, 5th ed. Washington DC: American Psychiatric Association; 2013. https://doi.org/10.1176/appi.books. 9780890425596

3. Berg KL, Shiu CS, Acharya K, Stolbach BC, Msall ME. Disparities in adversity among children with autism spectrum disorder: a population-based study. Developmental Medicine \& Child Neurology. 2016;58(11):1124-1131. doi 10.1111/dmcn.13161 https://doi.org/10.1111/dmcn.13161

4. Rigle B. The relationship between adverse childhood events, resiliency and health among children with autism. Journal of Autism and Developmental Disorders. 2017;47:187-202. doi 10.1007/s10803016-2905-3 https://doi.org/10.1007/s10803-016-2905-3

5. Kerns CM, Newschaffer CJ, Berkowitz SJ, Lee BK. Examining the association of autism and adverse childhood experiences in the national survey of children's health: The important role events of income and co-occurring mental health conditions. Journal of Autism and Developmental Disorders. 2017;47:2275-2281. doi: 10.1007/s10803-015-2392-y https://doi.org/10.1007/s10803-015-2392-y

6. Griffiths S, Allison C, Kenny R, Holt R, Smith P, Baron-Cohen S. The Vulnerability Experiences Quotient (VEQ): A study of vulnerability, mental health and life satisfaction in autistic adults. Autism Res. 2019(12):1516-1528. https://doi.org/10.1002/aur.2162

7. Rumball F. A systematic review of the assessment and treatment of post-traumatic stress disorder in individuals with autism spectrum disorders. Rev J Autism Dev Disord. 2019(6):294-324. https://doi. org/10.1007/s40489-018-0133-9

8. Lobregt-van Buuren E, Sizoo B, Mevissen L, De Jongh A. Eye Movement Desensitization and Reprocessing (EMDR) therapy as a feasible and potential effective treatment for adults with Autism Spectrum Disorder (ASD) and a history of adverse events. J Autism Dev Disord. 2019(49):51-164. https://doi.org/10.1007/s10803-018-3687-6

9. Copeland WE, Keeler G, Angold A, Costello J. Traumatic events and post-traumatic stress. Arch Gen Psychiatry. 2007;64(5):577-584. doi:10.1001/archpsyc.64.5.577 https://doi.org/10.1001/archpsyc. 64.5 .577

10. Bruin EI de, Ferdinand RF, Meester S, De Nijs, PFA,Verheij F. High rates of psychiatric co-morbidity in PDD-NOS. Journal of Autism and Developmental Disorders. 2007;7(37):877-886. https://doi. org/10.1007/s10803-006-0215-x

11. Hof van der B, Delorme R, Chaste P, Nyden A, Wentz E, Ståhlberg, O, et al. Psychiatric and psychosocial problems in adults with normal-intelligence autism spectrum disorders. BMC Psychiatry. 2009. http://www.biomedcentral.com/1471-244X/9/35 https://doi.org/10.1186/1471-244X-9-35 
12. Lugnegård TU, Hallerbäck MU, Gillberg C. Psychiatric comorbidity in young adults with a clinical diagnosis of Asperger Syndrome. Research in Developmental Disabilities. 2011;32:1910-1917. https://doi. org/10.1016/j.ridd.2011.03.025

13. Taylor JL, Gotham KO. Cumulative life events, traumatic experiences, and psychiatric symptomatology in transition-aged youth with autism spectrum disorder. Journal of developmental disorders. 2016;8:28. doi 10.1186/s11689-016-9160-y https://doi.org/10.1186/s11689-016-9160-y

14. Kildahl AN, Bakken TL, Iversen TE, Helverschou SB. Identification of post-traumatic stress disorder in individuals with autism spectrum disorder and intellectual disability: A systematic review. Journal of Mental Health Research in Intellectual Disabilities. 2019;12:1-2. doi.org/10.1080/19315864.2019. 1595233 https://doi.org/10.1080/19315864.2019.1595233

15. Klin A, Jones W, Schultz R, Volkmar F. The enactive mind, or from actions to cognition: lessons from autism. Philos Trans R Soc Lond B Biol Sci. 2003;358(1430):345-360. PM:12639332 https://doi. org/10.1098/rstb.2002.1202

16. De Jaegher H. Embodiment and sense-making in autism [Hypothesis and Theory]. Front Integr Neurosci, 2013;7(15). https://doi.org/10.3389/fnint.2013.00015 https://doi.org/10.3389/fnint.2013.00015

17. Crane L, Goddard L, Pring L. Sensory processing in adults with autism spectrum disorders. Autism, 2009;13(3):215-228. https://doi.org/10.1177/1362361309103794; https://doi.org/10.1177/ 1362361309103794

18. Roberts LA, Koenen KC, Lyall K, Robinson EB, Weisskopf MG. Association of autistic traits in adulthood with childhood abuse, interpersonal victimization and posttraumatic stress. Child Abuse Negl. 2015(45):135-142. https://doi.org/10.1016/j.chiabu.2015.04.010

19. Wood JJ, Gadow KD. Exploring the nature and function of anxiety in youth with autism spectrum disorders. Clin Psychol Sci Prac. 2010(17):281-292. https://doi.org/10.1111/j.1468-2850.2010.01220.x

20. Kerns CM, Newschaffer CJ, Berkowitz SJ. Traumatic childhood events and autism spectrum disorder. J Autism Dev Disord. 2015(45):3475-3486. https://doi.org/10.1007/s10803-015-2392-y

21. Spinazzola J, Blaustein M, Van der Kolk BA. Posttraumatic stress disorder treatment outcome research: the study of unrepresentative samples? Journal of Traumatic Stress. 2005;18(5):425-436. https://doi. org/10.1002/jts.20050

22. International Society for Traumatic Stress Studies. Posttraumatic Stress Disorder Prevention and Treatment Guidelines. Methodology and Recommendations. 2019. [Internet]. Available from: http:// www.istss.org/treating-trauma/new-istss-prevention-and-treatment-guidelines.aspx. [Accessed on 3rd May 2021].

23. Shapiro, F. Eye movement desensitization and reprocessing: Basic principles, protocols, and procedures. 2001 (2nd ed.). Guilford Press.

24. Mevissen L, Lievegoed R, Jongh, A De. EMDR treatment in people with mild ID and PTSD: 4 cases. Psychiatric Quarterly. 2011(82):43-57. https://doi.org/10.1007/s11126-010-9147-x

25. Mevissen, L, Didden, R, Korzilius, H, Jongh, A De. Eye movement desensitisation and reprocessing therapy for posttraumatic stress disorder in a child and an adolescent with mild to borderline intellectual disability: A multiple baseline across subjects study. Journal of applied research in intellectual disabilities, 2017;00:1-8, doi: 10.1111/jar.12335 https://doi.org/10.1111/jar.12335

26. Peterson JL, Earl RK, Fox EA, Ma R, Haidar G, Pepper M, et al. Trauma and Autism Spectrum Disorder: Review, Proposed Treatment Adaptations and Future Directions. J Child Adolesc Trauma. 2019(12):529-547. https://doi.org/10.1007/s40653-019-00253-5

27. Mevissen L, Didden R, Jongh A De. DITS-LVB Manual. Diagnostic Interview Trauma and Stressors Mild Mental Retardation. Accare Child Study Center. 2018. [Internet]. Available from: https://www. accare.nl/child-study-center/kennisdomein/dits-lvb [Accessed on 05 May 2021] 